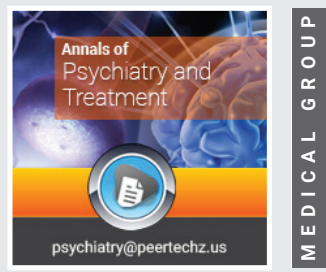

Research Article

\section{Clinical evidence in the phenomenon of Alien Abduction}

\section{Giulio Perrotta*}

Psychologist, Ph.D S., Forensic Criminologist, Legal Advisor sp.ed SSPL, Essayist, Institute for the study of psychotherapies - ISP, Via San Martino della Battaglia no. 31,00185 , Rome, Italy
Received: 02 November, 2021

Accepted: 20 December, 2021

Published: 21 December, 2021

*Corresponding author: Dr. Giulio Perrotta, Psychologist, Ph.D S., Forensic Criminologist, Legal Advisor sp.ed SSPL, Essayist, Institute for the study of psychotherapies - ISP, Via San Martino della Battaglia no. 31, 00185, Rome, Italy,

E-mail: info@giulioperrotta.com

ORCID: https://orcid.org/0000-0003-0229-5562

Keywords: Alien; Alien abduction; UFO; PICI-2; PDM-Q; PSM-Q; PAD-Q; PHEM

Copyright: (C) 2021 Perrotta G. This is an openaccess article distributed under the terms of the Creative Commons Attribution License, which permits unrestricted use, distribution, and reproduction in any medium, provided the original author and source are credited.

https://www.peertechzpublications.com

\title{
Abstract
}

Purpose: Starting from the classic definition of "alien abduction", the present research, starting from the study published in 2020 on the proposed clinical classification of this particular phenomenon, is aimed at confirming the theoretical assumption of psychopathological origin.

Methods: Clinical interview, based on narrative-anamnestic and documentary evidence and the basis of the Perrotta Human Emotions Model (PHEM) concerning their emotional and perceptual-reactive experience, and administration of the battery of psychometric tests published in international scientific journals by the author of this work: 1) Perrotta Integrative Clinical Interviews (PICl-2), to investigate functional and dysfunctional personality traits; 2) Perrotta Individual Sexual Matrix Questionnaire (PSM-Q), to investigate the individual sexual matrix; 3) Perrotta Affective Dependence Questionnaire (PAD-Q), to investigate the profiles of affective and relational dependence; 4) Perrotta Human Defense Mechanisms Questionnaire (PDM-Q), to investigate the defence mechanisms of the Ego.

Results: Preliminary results from the interviews and the anamnestic form would suggest that the phenomenon of alien abductions has a greater tendency to occur in the female group, in the adult and mature group (and tends to diminish but not disappear with advancing age) and in the group geographically originating in central-northern Italy (due to lower religious influences but greater openness to the typical contents of ufological and mystery narratives). Moreover, the subsequent results would lead us to deduce with almost total certainty, concerning the selected sample, that the phenomenon of alien abductions has an absolute prevalence in the believing population concerning the existence of paranormal phenomena per se, even in the absence of objective and/or scientific evidence. It is a phenomenon that is almost completely linked (110/112, 98.2\%) to a medium-low or not fully educated cultural level. Based on the $\mathrm{PICl}-2$ it emerged that the primary emerging disorder is alternatively the delusional disorder, the dissociative disorder and the narcissistic disorder; followed, as secondary disorders, by the delusional disorder (if it is not considered as primary disorder), the schizoid disorder, the borderline disorder, the obsessive disorder and the psychopathic disorder. The analysis of functional traits also reported the marked dysfunctional tendency of the classes referring to selfcontrol, sensitivity, Ego-Es comparison, emotionality, ego stability, security and relational functionality, confirming here too the marked dysfunctional tendency of the clinical population. According to the PSM-Q, almost $2 / 3$ of the participants $(73 / 112,65.2 \%)$ present a dysfunctional tendency to sexual behaviour and a marked tendency to chronicle feelings of shame in avoidance behaviour or hyposexuality. Furthermore, $100 \%$ of the sample of the population surveyed report having suffered significant or serious psychological or physical abuse at a young age, or intra-parental relational imbalances, or in any case a sexual upbringing that was not open and lacking in free communication. According to the PDM-Q, 27.7\% (31/112) are affected by affective dependency, with greater emphasis on types I (neurotic), VI (covert narcissist), V (borderline) and III (histrionic), in that order of descent. Finally, the PDM-Q reveals the widespread psychopathological tendency of the functional ego framework for the mechanisms of isolation, denial, regression, reactive formation, denial, projection, removal, withdrawal, instinct, repression and idealisation.

Conclusion: This research confirms the psychopathological nature of the alien abduction phenomenon, which deserves to be treated using a psychotherapeutic approach (preferably cognitive-behavioural and/or strategic) and possibly also pharmacological in serious cases, depending on the symptoms manifested and the severity of the morbid condition. 


\section{Introduction and background}

In the literature, the experience of alien abduction consists of the alleged kidnapping of human beings by extraterrestrial biological entities, using superior technologies. The information is not always clear whether these experiences are lived onboard spacecraft orbiting deep space outside the Earth's orbit or inside specially built underground military bases. The narratives supporting this circumstance are often different, as are the medical investigations that are carried out by an unspecified specialized technical staff [1].

Generally, in the field of ufology, encounters with creatures from other worlds and mysterious aircraft follow a precise identification, according to the indications of the astrophysicist Hynek and the Bloecher's directives [1]; in particular:

1. CE1 (type 1): One or more flying objects of defined solid mass or stray lights are sighted;

2. CE2 (type 2): There are phenomena caused by the presence of the flying object, such as the Crop Circles, the heat or radiation that damages the ground, human paralysis not otherwise diagnosed, interference with engines or radio-television reception and the loss of space-time cognition (intended as a flaw in the memory of a person associated with the alien encounter);

3. CE3 (type 3): Animated beings are seen, understood as extraterrestrial biological entities, in association with a sighting of unidentified flying objects;

4. CE4 (type 4): One witnesses or is the victim of alien abduction;

5. CE5 (type 5): There is a direct encounter with the extraterrestrial creature in a bilateral communication relationship established through conscious, voluntary and active human initiatives, or in any case through psychic cooperative communication;

6. CE6 (type 6): Long-term pathological effects are suffered, deriving from direct contact with the alien creature;

7. CE7 (type 7): There is finally the direct participation, the human-alien hybridization through mating or the birth of a creature that is the fusion with the terrestrial race.

In the scientific literature there are very few works dedicated to this topic, and all the studies published are focused in various ways on psychopathological profiles, albeit with population samples too small and therefore not sufficient to sanction the statistical representativeness; however, the data are still interested and deserve attention:

1. Research focuses attention on child abuse: Six adults, who had recently experienced sudden recall of preschool memories of sexual abuse or alien abduction/visitation, underwent comprehensive neuropsychological evaluations. All experiences "emerged" when hypnosis was used in a context of sexual abuse or New Age religion and were followed by a reduction in anxiety. As a group, these subjects showed significant elevations ( $\mathrm{T}$ greater than 70 ) of childhood imagery, complex partial epileptic signs, and suggestiveness. Neuropsychological data indicated right frontotemporal abnormalities and reduced access to the right parietal lobe. MMPI profiles were normal. The findings support the hypothesis that enhanced imagination due to temporal lobe lability within specific contexts may facilitate the creation of memories; they are further strengthened if there is also reduced anxiety [2].

2. Research focuses attention on sleeping disorders: It is clear that sleep is not only a global or cerebral phenomenon, but can also be a local phenomenon. This explains the fact that the primary states of being (wakefulness, NREM sleep, and REM sleep) are not necessarily mutually exclusive, and the components of these states can appear in various combinations, with fascinating clinical consequences. Examples include: sleep inertia, narcolepsy, sleep paralysis, lucid dreams, REM sleep behavior disorder, sleepwalking, sleep terrors, out-ofbody experiences, and reports of alien abduction. Incomplete state declaration also has implications for consciousness which also has fluid boundaries. Fluctuations in the degree of consciousness are likely explained by abnormalities in a "rhythm of spatial and temporal binding" that normally results in a unified conscious experience. Dysfunctional binding may play a role in anesthetic states, autism, schizophrenia, and neurodegenerative disorders. Further study of the broad spectrum of dissociated states of sleep and wakefulness that are closely related to states of consciousness and unconsciousness by basic neuroscientists, clinicians, and members of the legal profession will provide scientific, clinical, and therapeutic insights with forensic implications [3].

3. Research focuses attention on false memories: People sometimes fantasize entire complex scenarios and later define these experiences as memories of actual events rather than as imaginations. This article examines research associated with three such phenomena: past-life experiences, alien UFO contacts and abductions, and memory reports of childhood satanic ritual abuse. In each case, elicitation of fictional events is frequently associated with hypnotic procedures and structured interviews that provide strong, repeated requests for the requested experiences, and then legitimize the experiences as "real memories". Research associated with these phenomena supports the hypothesis that recollection is reconstructive and organized in terms of current expectations and beliefs $[4,5]$.

4. Research focuses attention on sleep paralysis: Sleep paralysis is a dissociative state that occurs primarily during waking and is characterized by alterations in motor, perceptual, emotional, and cognitive functions, such as the inability to perform voluntary movements, visual hallucinations feelings of pressure in the chest, delusions about a frightening presence, and, in some cases, fear of imminent death. Most people experience it infrequently, but typically when sleeping in the supine position; however, paralysis is considered an illness (parasonnia) when it is recurrent and/or associated with emotional burden. Interestingly, throughout human history, different peoples have interpreted paralysis according 
to a supernatural view. For example, Canadian Eskimos attribute paralysis to shaman spells, which hinder the ability to move and cause hallucinations of a formless presence. In Japanese tradition, paralysis is due to a vengeful spirit that suffocates its enemies during sleep. In Nigerian culture, a female demon attacks during a dream and causes paralysis. A modern manifestation of paralysis is the reporting of "alien abductions," experienced as an inability to move during waking associated with visual hallucinations of aliens. In all, paralysis is a significant example of how a specific biological phenomenon can be interpreted and shaped by different cultural contexts. To further explore the ethnopsychology of paralysis, in this review we present the "Pisadeira", a Brazilian folklore character native to the southeast of the country, but also present in other regions with variant names. The Pisadeira is described as a witch with long nails who lurks on rooftops at night and stomps on the chests of those who sleep with their stomachs full. This legend is mentioned in many anthropological accounts; however, no comprehensive references can be found about the Pisadeira from the perspective of sleep science [6]. Again, sleep paralysis accompanied by hypnopompic ("waking") hallucinations is an often frightening manifestation of the discordance between the cognitive/perceptual and motor aspects of Rapid Eye Movement (REM) sleep. Sleepers upon awakening become aware of an inability to move, and sometimes experience the intrusion of dream mentality into waking consciousness (e.g., seeing intruders in the bedroom). Two studies are summarized in this paper. In the first study, we evaluated 10 individuals who reported being abducted by space aliens and whose claims were linked to apparent episodes of sleep paralysis during which hypnopompic hallucinations were interpreted as alien beings. In the second study, adults reporting repressed, recovered, or ongoing memories of childhood sexual abuse more often reported sleep paralysis than a control group. Among the 31 who reported sleep paralysis, only one person linked it to memories of abuse. This person was among the six participants with recovered memory who reported sleep paralysis (i.e., $17 \%$ rate of interpreting it as related to abuse). People rely on personally plausible cultural narratives to interpret these otherwise puzzling episodes of sleep paralysis [7].

5. Research focuses attention on epileptic states and false memory: 20 male and 20 female undergraduate students were exposed singly for $20 \mathrm{~min}$. to an exotic setting (partial sensory deprivation and weak, bilateral transtemporal pulsed magnetic fields) that enhances relaxation and exotic experiences. The numerical incidence of subjective experiences described as old memories, dreams, emotions, or vestibular sensations did not differ significantly between the sexes; however, women who reported a greater prevalence of preexperimental complex partial epileptic-like signs were more likely to report experiences of "old memories" $(r=0.61)$ while men who exhibited these signs were more likely to report dream-like $(r=0.49)$ experiences. Because complex partial epileptic-like signs are positively associated with suggestibility, the potential contribution of this differential gender effect to the etiology of the False Memory Syndrome requires further investigation [8].

6. Research focuses attention on childhood sexual abuse: Twelve white middle-class women who had been severely sexually abused as children by a family member were asked to provide a narrative of their abuse and discuss their subsequent remembering and forgetting of these experiences. Most claimed they had undergone periods during which they had not recalled their abuse, but also claimed that they had never forgotten their experiences at another point during the interview. Nine of the women had actively tried to forget the abusive experiences, although 8 still experienced recurrent and often relentless intrusive memories. Our findings suggest that women with continuous memories may have longer and more coherent narratives than women without continuous memories. Implications of these findings for understanding the phenomenology of memory experiences and the concept of "recovered" memories of childhood sexual abuse are discussed [9]. People sometimes report recovering long-forgotten memories of childhood sexual abuse. The memory mechanisms that lead to such reports are not well understood, and the authenticity of recovered memories has often been questioned. We identified two subgroups of individuals reporting recovered memories of childhood sexual abuse. These subgroups differed dramatically in their cognitive profiles: People who recovered memories of abuse through suggestive therapy exhibited a greater susceptibility to the construction of false memories, but showed no tendency to underestimate their prior memories. In contrast, individuals who recovered memories of abuse spontaneously exhibited a greater propensity to forget their prior memories, but did not show a greater susceptibility to false memories. This double dissociation points to the mechanisms underlying recovered memory experiences and indicates that recovered memories may sometimes be fictitious and other times authentic $[10,11]$

In 2020, the writer tried to trace in specialized research all possible hypotheses that would explain the phenomena under consideration [12]. In particular, the following conclusions were reached [13-49]:

1. There is no documented evidence in the academic and scientific field about the chemical-physical investigations carried out on the findings extrapolated from humans, although there are suggestive reports from accredited laboratories about the mysterious origin of the same objects, with a strong indication for an extraterrestrial origin.

2. The patients who report having experienced alien abductions have a greater attraction and interest in paranormal phenomena and have a history of post-traumatic episodes and sleep paralysis (and disturbances), all events capable of generating false memories and episodes hallucinators, as in the hypothesis of post-operative awakening.

3. The strong tendency for patients to dissociate is connected with altered functionality of the temporal lobes.

4. The alien abductions, abuses with a satanic background and the identities of past lives seem connected to the reconstruction of false memories, typical in patients with high imagination and fantasy, connected to a hyperactivity of the upper longitudinal fasciculus and an altered functionality of the frontal lobe, of the prefrontal cortical regions, the 
hippocampal and vascular deficits (in particular, those referring to the anterior artery in the Willis's Polygon), in situations of high stress perceived by the patient.

5. The best treatment suggested in the scientific literature is the one combined between psychotherapy (strategic or cognitive-behavioural) and psychopharmacology (especially in the presence of evident clinical psychotic signs or disorders that need a pharmacological approach to stabilize the patient before continuing or setting up psychotherapy targeted and centred on the needs and needs specifically identified during the anamnesis). In the presence of confirmed dissociative episodes or rich production of false memories, it is not recommended to make use of hypnosis and suggestive techniques, which could incentivize or strengthen this patient's interpretation of reality.

6. The phenomenon of alien abduction, in the absence of scientific evidence, must be considered to have a psychopathological matrix. Therefore, a scale of severity of symptoms is identified (Perrotta Alien Abduction Scale, PAAS):

a) Level 1: Voluntary mystification.

b) Level 2: Delusions or hallucinations determined by the use of substances capable of altering the state of consciousness or by morbid neurological forms (such as epilepsy, tumours, vasculopathy, vascular disorders, trauma).

c) Level 3: False memories connected to post-traumatic stressful events, with pathologically oriented personality traits.

d) Level 4: Altered state caused by the obsessive idea of abduction, in comorbidity with sleep disturbances and pathologically oriented personality traits.

e) Level 5: Altered state caused by the dissociative disorder.

f) Level 6: Altered state caused by eccentric personality profiles (cluster B, DSM-V; cluster B, PICI-2).

g) Level 7: Altered state caused by psychotic personality profiles (cluster A, DSM-V; cluster C, PICI-2).

This theoretical approach appears, in the writer's opinion, to be complete and detailed, and will therefore be used in this research work as an evaluation scale concerning the symptoms manifested by the patients, part of the selected population.

\section{Research objectives and methods}

The methods used are two: 1) Clinical interview, based on narrative-anamnestic and documentary evidence and the basis of the Perrotta Human Emotions Model (PHEM) concerning their emotional and perceptual-reactive experience; 2) Administration of the battery of psychometric tests published in international scientific journals by the author of this work: a) Perrotta Integrative Clinical Interviews (PICI-2), to investigate functional and dysfunctional personality traits; b) Perrotta Individual Sexual Matrix Questionnaire (PSM-Q), to investigate individual sexual matrix; c) Perrotta Affective Dependence Questionnaire (PAD-Q), to investigate affective and relational dependence profiles; d) Perrotta Human Defense Mechanisms Questionnaire (PDM-O), to investigate ego defence mechanisms.

The phases of the research were divided as follows:

1) Selection of the population sample, according to the parameters indicated in the following paragraph.

2) Clinical interview, to each population group.

3) Administration of the Perrotta Integrative Clinical Interviews (PICI-2), Perrotta Individual Sexual Matrix Questionnaire (PSM-Q), Perrotta Affective Dependence Questionnaire (PAD-Q) e Perrotta Human Defense Mechanisms Questionnaire (PDM-Q).

4) Data processing following administration.

5) Comparison of data obtained.

\section{Setting and participants}

The requirements decided for the selection of the sample population are:

1) Age between 18 years and 67 years, healthy and robust constitution and in the absence of pathological symptoms: this choice is oriented to exclude underage subjects because not identified during the research of the sample population and subjects of mature or elderly age to avoid possible implications with neurodegenerative medical conditions and/ or neurovascular.

2) Italian nationality, with Italian ancestors in the last two generations: this choice is oriented in this sense to avoid cultural contaminations determined by popular beliefs different from the Christian-Catholic cult.

3) Precocious and manifest physical and psychic symptoms attributable to the state of alien abduction (altered perception of time with memory lapses, lucid dreams, bodily paralysis with fully or partially preserved state of consciousness, bodily signs and scars of unknown origin, perception of feeling observed or spied on, perceptions of metallic or distorted sounds in the absence of objective explanations, persistent insomnia, episodes of electromagnetic alteration to equipment present near the subject, physical fatigue, presence of bodily implants of unknown origin).

4) Declaration of alien possession based on the subjective experience, about the testimonies of family members.

5) Absence of psychopathological diagnosis or neurological and neurodegenerative disorder.

The selected setting, taking into account the protracted pandemic period (already in progress since the beginning of the present research), is the online platform via Skype and Video call Whatsapp, both for the clinical interview and for the administration. 
The present research work was carried out from March 2018 to September 2021. All participants were guaranteed anonymity and the ethical requirements of the Declaration of Helsinki are met.

Since the research is not financed by anyone, it is free of conflicts of interest.

The selected population clinical sample, which meets the requirements, is 112 participants, divided into five groups Tables 1,2.

\section{Results, limits and possible conflicts of interest}

After the selection of the chosen population sample (first stage), we proceeded with the clinical interviews (second stage), from which the first significant data emerged:

1) Considering the total population sample (112/112), one immediately notices the good disparity between the populousness of the overall female sample (67/112) compared to the male sample (45/112). Preliminary results from the interviews and the anamnestic form would suggest that the phenomenon of alien abductions has a greater tendency to occur in the female group, in the adult and mature group (and tends to diminish but not disappear with advancing age) and in the group geographically originating in central-northern Italy (due to lower religious influences but greater openness to the typical contents of ufological and mystery narratives) Moreover, the subsequent results would lead us to deduce with almost total certainty, concerning the selected sample, that the phenomenon of alien abductions has an absolute prevalence in the believing population concerning the existence of paranormal phenomena per se, even in the absence of objective and/or scientific evidence. It is a phenomenon almost completely linked $(110 / 112,98.2 \%)$ to a medium-low or not fully educated cultural level.

2) Also during the first clinical interview, other interesting data emerged that further strengthened the initial hypothesis:

a) All the proposed narratives presented fantastic and phantasmal elements, unsupported by evidentiary evidence

Table 1: Population sample (numerousness).

\begin{tabular}{|c|c|c|c|}
\hline Age & Male & Female & Total \\
\hline $18-27$ & 4 & 7 & 11 \\
\hline $28-37$ & 9 & 14 & 23 \\
\hline $38-47$ & 18 & 29 & 47 \\
\hline $48-57$ & 11 & 13 & 24 \\
\hline $58-67$ & 3 & 4 & 7 \\
\hline Total & $45(40.2 \%)$ & $67(59.8 \%)$ & $112(100 \%)$ \\
\hline
\end{tabular}

Table 2: Population sample (\% single category).

\begin{tabular}{|c|c|c|c|}
\hline Age & Male & Female & Total \\
\hline $18-27$ & $4(36.4 \%)$ & $7(63.6 \%)$ & $11(100 \%)$ \\
\hline $28-37$ & $9(39.1 \%)$ & $14(60.9 \%)$ & $23(100 \%)$ \\
\hline $38-47$ & $18(38.3 \%)$ & $29(61.7 \%)$ & $47(100 \%)$ \\
\hline $48-57$ & $11(45.8 \%)$ & $13(54.2 \%)$ & $24(100 \%)$ \\
\hline $58-67$ & $3(42.8 \%)$ & $4(57.2 \%)$ & $7(100 \%)$ \\
\hline
\end{tabular}

such as descriptive testimonies, photo-video-audio material and peculiar physical signs; 91/112 (81.2\%) claimed to possess unexplained bodily scars, without however supporting these claims with any specific clinical or material evidence.

b) At the clinical interviews, 48/112 (42.8\%) of the sample presented markedly psychotic symptoms (mainly paranoid, delusional, dissociative and schizophrenic) but denied ever having received a psychiatric diagnosis or having ever taken psychotropic drugs or having undergone psychotherapy.

3) Using, during the interview, the strategic language and the Perrotta Human Emotions Model (PHEM), it emerged that the totality of the selected population sample presents a full distress orientation, facilitating feelings such as guilt, shame, anger, fear and disappointment, in the presence of past (childhood) and current (interpersonal and work) family traumas. In fact, without the administration of questionnaires, it is evident that the impairment of perception in the plane of reality involves per se the manifestation of paranormal episodes described by patients, in the presence of a probable personality disorder to be identified with the help of psychodiagnostic tools.

The third stage of the research focused on the administration of the battery of questionnaires and these revealed the following results [50-108]

1) Administration of the Perrotta Integrative Clinical Interviews (PICI-2): Concerning the analysis of the dysfunctional traits (PICI-2TA), the primary disorder that emerged with at least 5 traits is delusional disorder $(60 / 112,53.6 \%)$, dissociative disorder (36/112, 32.1\%) and narcissistic disorder (18/112, 16\%); Secondary disorders include delusional disorder (if not considered as a primary disorder, 65/112, 58\%), schizoid disorder $(21 / 112,18.8 \%)$, borderline disorder (10/112, 8.9\%), obsessive disorder $(8 / 112,7.1 \%)$ and psychopathic disorder $(5 / 112,4.5 \%)$. A separate category that deserves specific mention is schizophrenic disorder, which was first diagnosed in $2 / 112(1.8 \%)$ of the population sample ( 1 woman and 1 man, both in the 38-47 age group) after the administration of the PICI-2, confirmed by the psychiatric examination proposed subsequently. In 108/112 (96.4\%) the following disorders emerged as comorbidities: ADHD (in the population under 27 years of age), body dysmorphism, ICT disorder, sleep disorders, eating disorders, anxiety and mood disorders, paraphiliac disorders and behavioural addictions (especially technology and internet addiction). The concrete suicide risk emerged in $23 / 112$ cases $(20.5 \%)$, while the presumed risk or request for attention emerged in $48 / 112(42.8 \%)$. Equally interesting is the cause/concause "epilepsy": $24 / 112(21.4 \%)$ report being on anti-epileptic therapy, while $71 / 112(63.4 \%)$ report having had in their lives at least one episode referable to epilepsy and/or a syncopal state of vasovagal origin. On the other hand, in the analysis of functional traits (PICI-2FT), it emerged that the most compromised classes, because they tended to be dysfunctional (with values of 0 or 4), were those referring to self-control, sensitivity, action, Ego-ID comparison, emotionality, ego stability, security and relational functionality, confirming here too the marked dysfunctional tendency of the clinical 
population. These findings place the clinical group under examination in correlation with the clinical group of subjects presumably affected by demonic possession. The preference for the administration of the PICI-2 concerning other widely validated and used psychometric tests, such as the MMPI-2, was for reasons of expediency: in fact, previous research has demonstrated the efficacy and efficiency, sometimes better indicated, of the PICI-2 concerning the MMPI-2, in terms of performance and completeness of diagnosis.

2) Administration of the Perrotta Individual Sexual Matrix Questionnaire (PSM-Q): The PSM questionnaires demonstrated that almost $2 / 3$ of the participants $(73 / 112,65.2 \%)$ show a dysfunctional tendency towards sexual behaviour and a marked tendency to chronicle feelings of shame in avoidance behaviour or hyposexuality. Furthermore, 100\% (112/112) of the sample of the population interviewed reported having suffered significant or serious psychological or physical abuse at a young age, or intra-parental relational imbalances, or in any case a sexual upbringing that was not open and lacking in free communication.

3) Administration of the Perrotta Affective Dependence Questionnaire (PAD-Q): According to the PDM-Q, 27.7\% (31/112) are affected by affective dependency, with greater emphasis on types I (neurotic), VI (covert narcissist), V (borderline) and III (histrionic), in this descending order.

4) Administration of the Perrotta Human Defense Mechanisms Questionnaire (PDM-Q): The PDM-Q reveals the widespread psychopathological tendency of the functional framework of the Ego, in the totality of the population sample (112/112) for the mechanisms of isolation, denial, regression, reactive formation, denial, projection, removal, withdrawal, instinct, repression and idealisation.

\section{Conclusion}

The last two steps served to reorder the results, and then draw conclusions. In particular:

1) Results obtained by Clinical interview: Preliminary results from the interviews and the anamnestic form would suggest that the phenomenon of alien abductions has a greater tendency to occur in the female group, in the adult and mature group (and tends to diminish but not disappear with advancing age) and in the group geographically originating in centralnorthern Italy (due to lower religious influences but greater openness to the typical contents of ufological and mystery narratives). Moreover, the subsequent results would lead us to deduce with almost total certainty, concerning the selected sample, that the phenomenon of alien abductions has an absolute prevalence in the believing population concerning the existence of paranormal phenomena per se, even in the absence of objective and/or scientific evidence. It is a phenomenon almost completely linked $(110 / 112,98.2 \%)$ to a medium-low or not fully educated cultural level.

2) Results were obtained by Perrotta Integrative Clinical Interviews (PICI-2): Based on the PICI-2 it emerged that the primary emerging disorder is alternately delusional disorder, dissociative disorder and narcissistic disorder; followed, as secondary disorders, by delusional disorder (if not considered as primary disorder), schizoid disorder, borderline disorder, obsessive disorder and psychopathic disorder. Also, the analysis of functional traits reported the marked dysfunctional tendency of the classes referring to self-control, sensitivity, Ego-Es comparison, emotionality, ego stability, security and relational functionality, confirming here the marked dysfunctional tendency of the clinical population.

3) Results were obtained by Perrotta Individual Sexual Matrix Questionnaire (PSM-Q): According to the PSM-Q almost 2/3 of the participants $(73 / 112,65.2 \%)$ show a dysfunctional tendency towards sexual behaviour and a marked tendency to chronicle feelings of shame into avoidance behaviour or hyposexuality. Again, $100 \%$ of the sample of the population surveyed report having suffered significant or serious psychological or physical abuse at a young age, or intra-parental relational imbalances, or in any case a sexual upbringing that was not open and lacking in free communication.

4) Results were obtained by Perrotta Affective Dependence Questionnaire (PAD-Q): According to the PDM-Q, 27.7\% (31/112) are affected by affective dependency, with greater emphasis on types I (neurotic), VI (covert narcissist), V (borderline) and III (histrionic), in this descending order.

5) Results were obtained by Perrotta Human Defense Mechanisms Questionnaire (PDM-Q): The PDM-Q reveals the widespread psychopathological tendency of the functional ego framework for the mechanisms of isolation, denial, regression, reactive formation, denial, projection, removal, withdrawal, instinct, repression and idealisation.

In conclusion, this research confirms the psychopathological nature of the alien abduction phenomenon, which deserves to be treated using a psychotherapeutic approach (preferably cognitive-behavioural and/or strategic) [109] and possibly also pharmacological in serious cases, depending on the symptoms manifested and the severity of the morbid condition.

\section{References}

1. Perrotta G (2017) Criminologia esoterica. 2nd ed. Primiceri Ed.

2. Persinger MA (1992) Neuropsychological profiles of adults who report "sudden remembering" of early childhood memories: implications for claims of sex abuse and alien visitation/abduction experiences. Percept Mot Skills 75: 259-266. Link: https://bit.ly/3sladfa

3. Mahowald MW, Cramer Bornemann MA, Schenck CH (2011) State dissociation, human behavior, and consciousness. Curr Top Med Chem 11 2392-2402. Link: https://bit.ly/3qfPIOB

4. Spanos NP, Burgess CA, Burgess MF (1994) Past-life identities, UFO abductions, and satanic ritual abuse: the social construction of memories. In J Clin Exp Hypn 42: 433-446. Link: https://bit.ly/3GSYQ20

5. de Sa JFR, Mota-Rolim SA (2016) Sleep Paralysis in Brazilian Folklore and Other Cultures: A Brief Review. Front Psychol 7: 1294. Link: https://bit.ly/3E9q02Z

6. McNally RJ, Clancy SA (2005) Sleep paralysis, sexual abuse, and space alien abduction. Transcult Psychiatry 42: 113-122. Link: https://bit.ly/3J6PCkC

7. Persinger MA (1994) Elicitation of "childhood memories" in hypnosis-like 
settings is associated with complex partial epileptic-like signs for women but not for men: implications for the false memory syndrome. Percept Mot Skills 78: 643-651. Link: https://bit.ly/3FgUK3m

8. Fivush R, Edwards VJ (2004) Remembering and forgetting childhood sexual abuse. J Child Sex Abus 13: 1-19. Link: https://bit.ly/32mG09p

9. Geraerts E, Lindsay DS, Merckelbach H, Jelicic M, Raymaekers L, et al (2009) Cognitive mechanisms underlying recovered-memory experiences of childhood sexual abuse. Psychol Sci 20: 92-98. Link: https://bit.ly/3sqIPwB

10. Geraerts E (2012) Cognitive underpinnings of recovered memories of childhood abuse. Nebr Symp Motiv 58: 175-191. Link: https://bit.ly/3FdxRhx

11. Coons PM (1994) Reports of satanic ritual abuse: further implications about pseudomemories. Percept Mot Skills 78: 1376-1378. Link: https://bit.ly/3e924SN

12. Perrotta G (2020) Alien Abduction Experience: definition, neurobiological profiles, clinical contexts and therapeutic approaches. Ann Psychiatry Treatm 4: 025-029. Link: https://bit.ly/3J6MqWs

13. French C, Holden KJ (2002) Alien abduction experiences: some clues from neuropsychology and neuropsychiatry. Cognitive Neuropsychiatry 7: 163178. Link: https://bit.ly/3ylrFLL

14. Forrest DV (2008) Alien abduction: a medical hypothesis. J Am Acad Psychoanal Dyn Psychiatry 36: 431-442. Link: https://bit.ly/30HFVla

15. Spanos NP, BurgessCA, Burgess MF (1994) Past-life identities, UFO abductions, and satanic ritual abuse: the social construction of memories. Int J Clin Exp Hypn. 42: 433-446. Link: https://bit.ly/3GSYQ20

16. O'Mara S (2009) Torturing the brain. Trend in Cognitive Sciences 13: 497-500. Link: https://bit.ly/3qeKCSm

17. McNally RJ, Lasko NB, Clancy SA, Macklin ML, Pitman RK et al. (2004) Psychophysiological responding during script-driven imagery in people reporting abduction by space aliens. Psychol Sci 15: 493-497. Link: https://bit.ly/3ssWrHk

18. Appelle S, Lynn SJ, Newman LS (2012) Alien abduction experiences. Link: https://bit.ly/3eaQFIm

19. Paley J (2007) Satanic abuse and alien abduction: a comparative analysis theorizing temporal lobe activity as a possible connection between anomaloous memories. Bri J Social Work 27: 43-70. Link: https://bit.ly/3ylK6A6

20. Perrotta G (2019) Psicologia clinica, 1st ed, Luxco Ed.

21. Perrotta G (2019) The reality plan and the subjective construction of one's perception: the strategic theoretical model among sensations, perceptions, defence mechanisms, needs, personal constructs, beliefs system, social infl uences and systematic errors. J Clin ResRep 1. Link: https://bit.ly/38Ztg3S

22. Malanga C (1998) Gli UFO nella mente. Interferenze aliene. Bompiani, Milano.

23. Malanga C (2010) Alieni o Demoni, Terre Sommerse Ed.

24. Malanga C (2010) Alien Cicatrix. P. 1-2; E-book Ed.

25. Roger KL (2005) The Aliens and the Scalpel: Scientific Proof of Extraterrestria Implants in Humans, Book Tree.

26. Irwin H, Schofield M, Baker IS (2014) Dissociative tendencies, sensory processing sensitivity and aberrant salience as predictors of anomalous experiences and paranormal attributions. J Society Psychical Res 78: 193206. Link: https://bit.ly/3E7fMAl

27. Holden KJ, French C (2002) Alien abduction experiences: some clues from neuropsychology and neuropsychiatry. Cogn Neuropsychi 7: 163-178. Link: https://bit.ly/3ylrFLL

28. Bartholomew RE, Keith B, George SH (1991) UFO abductees and contactees: Psychopathology or fantasy proneness? Professional Psychology: Research and Practice 22: 215-222. Link: https://bit.ly/32cEe6m

29. Cheyne JA (2001) The ominous numinous: Sensed presence and 'other' hallucinations. J Consciousnes Studies 8: 133-150. Link: https://bit.ly/3p8yQKa
30. Cheyne JA, Newby-Clark IR, Rueffer SD (1999) Relations among hypnagogic and hypnopompic experiences associated with sleep paralysis. J Sleep Res 8: 313-317. Link: https://bit.ly/3E60pWY

31. Hobson JA, McCarley RW (1997) The brain as a dream state generator: An activationsynthesis hypothesi s of the dream process. Am J Psychiatry 134 1335-1348. Link: https://bit.ly/30HcRAw

32. Newman LS, Baumeister RF (1996) Toward an explanation of the UFO abduction phenomenon: Hypnotic elaboration, extraterrestrial sadomasochism, and spurious memories. Psychological Inquir 7: 99-126. Link: https://bit.ly/3yQ07mc

33. Persinger MA, Makarec K (1987) Temporal lobe epileptic signs and correlative behaviors displayed by normal populations. J General Psychology 114: 179195. Link: https://bit.ly/3qupF6r

34. Persinger MA, Tiller SG, Koren SA (2000) Experimental simulation of a haunt experience and elicitation of paroxysmal electroencephalographi c activity by transcerebral complex magnetic fields: Induction of a synthetic 'ghost'? Percept Mot Skills 90: 659-674. Link: https://bit.ly/3yHuDAh

35. Persinger MA, Valliant PM (1985) Temporal lobe signs and reports of subjective paranormal experiences in a normal population. Percept Mot Skills 60: 903-909. Link: https://bit.ly/33Lf0wn

36. Powers SM (1994) Dissociation in alleged extraterrestrial abductees Dissociation 7: 44-50. Link: https://bit.ly/3sIS2Go

37. Remillard GM, Andermann F, Testa GF, Gloor P, Aube M, et al. (1983) Sexual ictal manifestations predominate in women with temporal lobe epilepsy: a finding suggesting sexual dimorphism in the human brain. Neurology 33 : 323-330. Link: https://bit.ly/3mjgi8d

38. Ring K (1990) The Omega Project: A psychologica I survey of persons reporting abductions and other UFO encounters. J UFO Studies 2: 59-98.

39. Rodeghier M (1991) Psychosocial characteristics of abductees: Results from the CUFOS abduction project. J UFO Studies 3: 59-90.

40. Schacter DL, Reiman E, Curran T, Yun LS, Bandy D, et al. (1996) Neuroanatomica I correlates of veridical and illusory recognition memory: Evidence from positron emission tomography. Neuron 17: 267-274. Link: https://bit.ly/3Eb9zmY

41. Symons D (1993) The stuff that dreams aren't made of: Why wake-state and dream-state sensory experiences differ. Cognition 47: 181-217. Link: https://bit.ly/3e6QOGH

42. Terrillon JC, Marques-Bonham S (2001) Does recurrent isolated sleep paralysis involve more than cognitive neurosciences? Journal of Scientific Explanation 15: 97-123. Link: https://bit.ly/3J60Nly

43. Twemlow SW, Gabbard GO, Jones FC (1982) The out-of-body experience: A phenomenologica I typology based on questionnaire responses. Am J Psychiatry 139: 450-455. Link: https://bit.ly/3paZLF2

44. French C, Santomauro J, Hamilton V, Fox R, Thalbourne MA (2008) Psychological aspects of the alien contact experience. Cortex 44: 1387-1395. Link: https://bit.ly/3JgBshh

45. Forrest DV (2008) Alien abduction: a medical hypothesis. J Am Acad Psychoanal Dyn Psychiatry 36: 431-442. Link: https://bit.ly/30HFVla

46. O'Mara S (2009) Torturing the brain: on the folk psychology and folk neurobiology motivating 'enhanced and coercive interrogation techniques'. Trends Cogn Sci 13: 497-500. Link: https://bit.ly/3smioYU

47. McNally RJ, Lasko NB, Clancy SA, Macklin ML, Pitman RK et al. (2004) Psychophysiological responding during script-driven imagery in people reporting abduction by space aliens. Psychol Sci 15: 493-497. Link: https://bit.ly/3ssWrHk

48. Appelle S (2012) Alien abduction experiences. Research Gate. PsycINFO Database Record.

49. Paley J (2007) Satanic abuse and alien abduction: a comparative analysis theorizing temporal lobe activity as a possible connection between anomaloous memories. Bri J Social Work 27: 43-70. Link: https://bit.ly/3ylK6A6 
50. Perrotta G (2020) The strategic clinical model in psychotherapy: theoretical and practical profiles. J Addi Adol Behav 3: 5. Link: https://bit.ly/3aPMx9X

51. Perrotta G (2020) Accepting "change" in psychotherapy: from consciousness to awareness. J Addict Res Adolesc Behav 3.

52. Perrotta G (2021) The "Human Emotions" and the "Perrotta Human Emotions Model" (PHEM): The new theoretical model. Historical, neurobiological and clinical profiles. Arch Depress Anxiety 7: 020-027. Link: https://bit.ly/3ylf8rO

53. Perrotta G (2020) Psychological trauma: definition, clinical contexts, neural correlations and therapeutic approaches. Curr Res Psychiatry Brain Disord: CRPBD-100006. Link: https://bit.ly/37UD3bz

54. Perrotta G (2020) Dysfunctional attachment and psychopathological outcomes in childhood and adulthood. Open J Trauma 4: 012-021. Link: https://bit.ly/2Mi2ThB

55. Perrotta G (2020) Neonatal and infantile abuse in a family setting. Open $J$ Pediatr Child Health 5: 034-042. Link: https://bit.ly/2KApVQo

56. Perrotta G (2021) Parental Alienation Syndrome (PAS): definition, humanistic profiles and clinical hypothesis of absorption with "adaptation disorder". Clinical evidence. Open J Pediatr Child Health 6: 026-035. Link: https://bit.ly/3yJbJsD

57. Perrotta G, Fabiano G (2021) Behavioural disorders in children and adolescents: Definition, clinical contexts, neurobiological profiles and clinical treatments. Open J Pediatr Child Health 6: 005-015. Link: https://bit.ly/32gWTxt

58. Perrotta G (2019) The reality plan and the subjective construction of one's perception: the strategic theoretical model among sensations, perceptions, defence mechanisms, needs, personal constructs, beliefs system, social influences and systematic errors. J Clin Res Rep 1. Link: https://bit.ly/38Ztg3S

59. Perrotta G (2019) Delusions, paranoia and hallucinations: definitions, differences, clinical contexts and therapeutic approaches. J Neurol (CJNE) 1 : 22-28. Link: https://bit.ly/3J6VoCU

60. Perrotta G (2021) The state of consciousness: from perceptual alterations to dissociative forms. Defining, neurobiological and clinical profiles. J Neuro Neurol Sci Disord 7: 006-018. Link: https://bit.ly/3mjxBpP

61. Perrotta G (2020) The structural and functional concepts of personality: The new Integrative Psychodynamic Model (IPM), the new Psychodiagnostic Investigation Model (PIM) and the two clinical interviews for the analysis of personality disorders (Perrotta Integrative Clinical Interview or $\mathrm{PICl}$ ) for adults and teenagers (1TA version) and children (1C version). Psychiatry Peertechz, E-book. Link: https://bit.ly/3GXWL4X

62. Perrotta G (2020) First revision of the Psychodiagnostic Investigation Mode (PIM-1R) and elaboration proposal of a clinical interview for the analysis of personality disorders (Perrotta Integrative Clinical Interview or $\mathrm{PICl}$ 1) for adults, teenagers and children. Psychiatry Peertechz, E-book. Link: https://bit.ly/3mjJsnF

63. Perrotta G (2020) Perrotta Integrative Clinical Interview (PICl-1): Psychodiagnostic evidence and clinical profiles in relation to the MMPI-II. Ann Psychiatry Treatm 4: 062-069. Link: https://bit.ly/3yleR8g

64. Perrotta G (2021) “Perrotta Integrative Clinical Interview” (PICl) for adults and teenagers (1TA version) and children (1C version): new theoretical models and practical integrations between the clinical and psychodynamic approach Ann Psychiatry Treatm 5: 001-014. Link: https://bit.ly/3yGsYep

65. Perrotta G (2021) Perrotta Integrative Clinical Interview (PICl-1): a new revision proposal for PICl-1TA. Two single cases. Glob J Medical Clin Case Rep 8: 041-049. Link: https://bit.ly/30LPK86

66. Perrotta G (2021) Perrotta Integrative Clinical Interviews (PICl-2): innovations to the first model, the study on the new modality of personological investigation, trait diagnosis and state diagnosis, and the analysis of functional and dysfunctional personality traits. An integrated study of the dynamic, behavioural, cognitive and constructivist models in psychopathological diagnosis. Ann Psychiatry Treatm 5: 067-083. Link: https://bit.ly/3pegEyA
67. Perrotta G (2019) Anxiety disorders: definitions, contexts, neural correlates and strategic therapy. J Neurol Neurosci 6: 046. Link: https://bit.ly/3Eh9WMG

68. Perrotta G (2019) Neural correlates in eating disorders: Definition, contexts and clinical strategies. J Pub Health Catalog 2: 137-148. Link: https://bit.ly/3FhoZHI

69. Perrotta G (2019) Post-traumatic stress disorder: Definition, contexts, neura correlations and cognitive-behavioural therapy. J Pub Health Catalog 2: 4047. Link: https://bit.ly/3J0IOGF

70. Perrotta G (2019) Sleep-wake disorders: Definition, contexts and neural correlations. J Neurol Psychol 7: 09. Link: https://bit.ly/3yMdjKn

71. Perrotta G (2019) Tic disorder: definition, clinical contexts, differential diagnosis, neural correlates and therapeutic approaches. J Neurosci Rehab 2019: 1-6. Link: https://bit.ly/3Eb8R9i

72. Perrotta G (2019) Depressive disorders: Definitions, contexts, differentia diagnosis, neural correlates and clinical strategies. Arch Depress Anxiety 5 : 009-033. Link: https://bit.ly/3sI93Az

73. Perrotta G (2019) Panic disorder: definitions, contexts, neural correlates and clinical strategies. Curr Tr Clin Med Sci 1: 2019. Link: https://bit.ly/3EcJ5Bi

74. Perrotta G (2019) Obsessive-Compulsive Disorder: definition, contexts, neural correlates and clinical strategies. J Neurol 1: 08-16. Link: https://bit.ly/3yGFZoe

75. Perrotta G (2019) Behavioral addiction disorder: definition, classifications, clinical contexts, neural correlates and clinical strategies. J Addi Adol Beh 2. Link: https://bit.ly/3GVtMPm

76. Perrotta G (2019) Paraphilic disorder: definition, contexts and clinica strategies. Neuro Research 1: 4. Link: https://bit.ly/3Eefg3B

77. Perrotta G (2019) Internet gaming disorder in young people and adolescent: a narrative review. J Addi Adol Beh 2.

78. Perrotta G (2019) Bipolar disorder: definition, differential diagnosis, clinical contexts and therapeutic approaches. J Neuroscience and Neurological Surgery 5. Link: https://bit.ly/33LObda

79. Perrotta G (2020) Suicidal risk: definition, contexts, differential diagnosis, neural correlates and clinical strategies. J Neuroscience and Neurological Surgery 6: 114. Link: https://bit.ly/3Fg6RO6

80. Perrotta G (2020) Pathological gambling in adolescents and adults: definition, clinical contexts, differential diagnosis, neural correlates and therapeutic approaches. ES J Neurol 1: 1004. Link: https://bit.ly/3FeWmuE

81. Perrotta G (2020) Pedophilia: definition, classifications, criminological and neurobiological profiles and clinical treatments. A complete review. Open $J$ Pediatr Child Health 5: 019-026. Link: https://bit.ly/3eaPL8t

82. Perrotta G (2020) The concept of altered perception in "body dysmorphic disorder": the subtle border between the abuse of selfies in social networks and cosmetic surgery, between socially accepted dysfunctionality and the pathological condition. J Neurol Neurol Sci Disord 6: 001-007. Link: https://bit.ly/3qfOvXz

83. Perrotta G (2020) Sexual orientations: a critical review of psychological clinical and neurobiological profiles. The clinical hypothesis of homosexual and bisexual positions. Int $\mathrm{J}$ Sex Reprod Health Care 3: 027-041. Link: https://bit.ly/3J57bSf

84. Perrotta G (2020) Borderline Personality Disorder: definition, differential diagnosis, clinical contexts and therapeutic approaches. Ann Psychiatry Treatm 4: 043-056. Link: https://bit.ly/3pcAqLa

85. Perrotta G (2020) Narcissism and psychopathological profiles: definitions, clinical contexts, neurobiological aspects and clinical treatments. J Clin Cases Rep 4: 12-25. Link: https://bit.ly/3yl4LnO

86. Perrotta G (2020) Dysfunctional sexual behaviours: definition, clinical contexts, neurobiological profiles and treatments. Int J Sex Reprod Health Care 3: 061-069. Link: https://bit.ly/3spkjM6

87. Perrotta G (2020) Bisexuality: definition, humanistic profiles, neural correlates 
and clinical hypotheses. J Neuroscience and Neurological Surgery 6. Link: https://bit.ly/3sl6f6h

88. Perrotta G (2021) Histrionic personality disorder: Definition, clinical profiles, differential diagnosis and therapeutic framework. Arch Community Med Public Health 7: 001-005. Link: https://bit.ly/3p9TAkL

89. Perrotta G (2020) Affective Dependence: from pathological affectivity to personality disorders. Definitions, clinical contexts, neurobiological profiles and clinical treatments. Health Sci 1: 1-7. Link: https://bit.ly/3ebySL3

90. Perrotta G (2020) Psychotic spectrum disorders: definitions, classifications, neural correlates and clinical profiles. Ann Psychiatry Treatm 4: 070-084. Link: https://bit.ly/3FdnOsx

91. Perrotta G (2021) Maladaptive stress: Theoretical, neurobiological and clinical profiles. Arch Depress Anxiety 7: 001-007. Link: https://bit.ly/32lgl6F

92. Perrotta G (2021) Sexual fantasies: the boundary between physiology and psychopathology. Int J Sex Reprod Health Care 4: 042-052. Link: https://bit.ly/324B3h7

93. Perrotta G (2021) Clinical evidence in sexual orientations: definitions, neurobiological profiles and psychological implications. Ann Psychiatry Treatm 5: 043-053. Link: https://bit.ly/3EjFEco

94. Perrotta G (2021) "Polygamous perception" and couple's relational choice: definitions, socio-cultural contexts, psychopathological profiles and therapeutic orientations. Clinical evidence. Ann Psychiatry Treatm 5: 054061. Link: https://bit.ly/3e8YCYI

95. Perrotta G (2021) The learning of specific dysfunctional behavioural patterns through social network and telematics platforms in preadolescents and adolescents. Psychopathological clinical evidence. Open J Pediatr Child Health 6: 026-035. Link: https://bit.ly/3EcnZ6f

96. Perrotta G (2020) Epilepsy: from pediatric to adulthood. Definition, classifications, neurobiological profiles and clinical treatments. J Neurol Neurol Sci Disord 6: 014-029. Link: https://bit.ly/3J4mmex

97. Perrotta G (2020) The pharmacological treatment of epileptic seizures in children and adults: introduction, clinical contexts, psychopharmacological profiles and prospects in the neurogenetic field. Journal of Neuroscience and Neurological Surgery 6. Link: https://bit.ly/3e8Y9W5
98. Perrotta G (2021) Avoidant personality disorder: Definition, clinical and neurobiological profiles, differential diagnosis and therapeutic framework. $J$ Neuro Neurol Sci Disord 7: 001-005. Link: https://bit.ly/3yPIHYv

99. Perrotta G (2021) Clinical evidence in Troilism (Polygamy and Polyamory): definition, psychological profiles and clinical implications. Int J Sex Reprod Health Care 4: 073-079. Link: https://bit.ly/3mjeLyZ

100. Perrotta G (2021) The diagnosis of personality traits in "affective dependency": when the toxic bond is an expression of a personality disorder. Research. Int J Sex Reprod Health Care 4: 085-090. Link: https://bit.ly/3J6Uk1S

101.Perrotta G (2021) Massive use of tattoos and psychopathological clinical evidence. Arch Community Med Public Health 7: 079-085. Link: https://bit.ly/3p8wyuy

102. Perrotta G (2021) Clinical evidence in the phenomenon of demoniac possession. Ann Psychiatry Treatm 5: 088-095. Link: https://bit.ly/3mgeKLZ

103. Perrotta G (2021) Perrotta Individual Sexual Matrix Questionnaire (PSM 1). The new clinical questionnaire to investigate the main areas of the individual sexual matrix. Int J Sex Reprod Health Care 4: 013-021. Link: https://bit.ly/3H1oLVh

104.Perrotta G (2021) Perrotta Individual Sexual Matrix Questionnaire (PSM-Q): Technical updates and clinical research. Int J Sex Reprod Health Care 4: 062 066. Link: https://bit.ly/3FgcllL

105. Perrotta G (2021) Perrotta Affective Dependence Questionnaire (PAD-Q) Clinical framing of the affective-sentimental relational maladaptive model. Ann Psychiatry Treatm 5: 062-066. Link: https://bit.ly/324Ae81

106. Perrotta G (2021) Perrotta Affective Dependence Questionnaire (PAD-Q): Psychodiagnostic evidence and clinical profiles. Int J Sex Reprod Health Care 4: 080-084. Link: https://bit.ly/3e8WrUF

107. Perrotta G (2020) Human mechanisms of psychological defence: definition historical and psychodynamic contexts, classifications and clinical profiles. Int J Neurorehabilita Eng 7: 1000360. Link: https://bit.ly/3sIMnQK

108. Perrotta G (2021) Perrotta Human Defense Mechanisms Questionnaire (PDM-Q): The new psychodiagnostic tool to identify human psychological defense mechanisms and their clinical implications. Arch Depress Anxiety 7 029-033. Link: https://bit.ly/3Ecql54

109. Perrotta G (2021) Strategic psychotherapy and the "decagonal model" in clinical practice. Ann Psychiatry Treatm 5: 028-035. Link:

\section{Discover a bigger Impact and Visibility of your article publication with}

\section{Peertechz Publications}

\author{
Highlights \\ * Signatory publisher of ORCID \\ * Signatory Publisher of DORA (San Francisco Declaration on Research Assessment) \\ * Articles archived in worlds' renowned service providers such as Portico, CNKI, AGRIS, \\ TDNet, Base (Bielefeld University Library), CrossRef, Scilit, J-Gate etc. \\ - Journals indexed in ICMJE, SHERPA/ROMEO, Google Scholar etc. \\ * OAI-PMH (Open Archives Initiative Protocol for Metadata Harvesting) \\ * Dedicated Editorial Board for every journa \\ * Accurate and rapid peer-review process \\ * Increased citations of published articles through promotions \\ * Reduced timeline for article publication \\ Submit your articles and experience a new surge in publication services \\ (https://www.peertechz.com/submission).
}

